\title{
Registros taxonômicos de cestóides Trypanorhynchal Homeacanthoidea em elasmobrânquios coletados na costa do Estado do Paraná, Brasil
}

\author{
MARCELO KNOFF*, SÉRGIO CARMONA DE SÃO CLEMENTE**, \\ ROBERTO MAGALHÃES PINTO* e DELIR CORRÊA GOMES*
}

\section{TAXONOMIC REPORTS OF TRYPANORHYNCHA/HOMEACANTHOIDEA CESTODES IN ELASMOBRANCHS COLLECTED IN THE COAST OF THE STATE OF PARANÁ, BRAZIL}

Specimes of elasmobranch fishes, captured in 1998 in the the State of Paraná, Brazil, were parasitized with Trypanorhyncha/Homeacanthoidea cestodes: Carcharhinus signatus with Heteronybelinia yamagutii (Dollfus, 1960) Palm, 1999 and H. nipponica (Yamaguti, 1952) Palm, 1999; Dipturus trachydermus (Krefft \& Stehmann, 1975) with Mixonybelinia beveridgei (Palm et al, 1997) Palm, 1999. Details of scolex morphology of the two species of Heteronybelinia that expanded their desciptions were obtained. The known geographical distribution of the species is widened.

Key words: Trypanorhyncha, Homeacanthoidea, Taxonomic reports, Elasmobranchs, Brazil.

\section{INTRODUÇÃO}

A presente pesquisa inclui um estudo taxonômico, com discussão de cestóides Trypanorhyncha/Homeacanthoidea parasitos de elasmobrânquios, coletados no litoral do estado do Paraná, onde foram determinadas as espécies das formas pós-larvares encontradas.

Das quinze espécies existentes no gênero Heteronybelinia, apenas três apresentam a armadura dos ganchos da região basal característica, contendo ganchos de formato digitiformes ${ }^{1-4}$, exatamente como as duas espécies pertencentes a este gênero coletadas neste trabalho.

Heteronybelinia yamagutii encontrada parasitando Carcharhinus signatus, teve a sua descrição original feita a partir de uma pós-larva registrada da cavidade celomática do peixe teleósteo Liosaccus cutaneus, na costa de Dakar, Senegal ${ }^{2}$. Outros registros desta espécie estão relacionados a moluscos cefalópodes do Atlântico Norte $^{5-7}$, de um cefalópode e seis teleósteos coletados da costa da Africa ${ }^{8}$. Palm revisando material depositado no British Museum of Natural History, Londres., registra as medidas de um espécime adulto coletado do estômago de Sphyrna lewini da Africa do $\mathrm{Sul}^{4}$.

* Laboratório de Helmintos Parasitos de Vertebrados, Departamento de Helmintologia, Instituto Oswaldo Cruz. Avenida Brasil 4365, CEP 21045-900, Rio de Janeiro, RJ, Brasil. E-mail: knoffm@ ioc.fiocruz.br

** Faculdade de Veterinária, Universidade Federal Fluminense, Rua Vital Brazil 64, CEP 24230-340, Niterói, RJ, Brasil. 
As pós-larvas de Heteronybelynia nipponica, encontradas em $C$. signatus no nosso litoral, tem como hospedeiro tipo Neobithytes macrops, também sendo registrada para Argentina kagoshimae, Xystrias grigorjewi (= Eopsetta grigorjewi) e Pseudorhombus pentophthalmus peixes teleósteos do Japão ${ }^{1}$.

Mixonybelinia beveridgei coletada em nosso litoral em Dipturus trachydermus, foi descrita a partir de pós-larvas encontradas em peixes teleósteos do continente africano, Chascanopsetta lugubris, Caelorinchus braueri e Lophiodes mutilus de Moçambique, e de Caelorinchus fasciatus da Namibia ${ }^{8}$.

\section{MATERIAL E MÉTODOS}

Em março de 1998, 13 peixes elasmobrânquios, 5 (1 fêmea e 4 machos) C. signatus (120$150 \mathrm{~cm} \mathrm{ct}$ ) e 8 ( 1 fêmea e 7 machos) $D$. trachydermus $(133.5-176 \mathrm{~cm} \mathrm{ct})$ foram capturados a, aproximadamente, 125 milhas do litoral do Estado do Paraná (25 50 'S $25^{\circ} 52^{\prime} \mathrm{S}$ e $45^{\circ} 23^{\prime} \mathrm{O}-45^{\circ} 25^{\prime} \mathrm{O}$; 200-500 $\mathrm{m}$ de profundidade), com espinhel de fundo. A bordo, seus estômagos e válvulas espirais foram retirados, etiquetados e preservados em gelo, antes de serem examinados. Os cestóides Trypanorhyncha foram coletados, fixados, corados e montados ${ }^{9}$. A organização sistemática dos Trypanorhyncha baseou-se em Campbell \& Beveridge ${ }^{10}$. Sinônimos são fornecidos para espécies que não foram tratadas por Dollfus ${ }^{11}$. As medidas dos escólex e proglotes e as terminologias utilizadas, seguem outros ${ }^{4,10,11}$.
Todas as medidas estão indicadas em milímetros (mm); a média (quando indicada) é apresentada seguida da amplitude de variação, entre parênteses. No sumário taxonômico são indicados o total de espécimes parasitos coletados e a infrapopulação de cada hospedeiro. Nome de hospedeiros que representam novos registros para a espécie parasita são seguidos de "NH" e/ou por "NDG" quando representar ampliação da distribuição geográfica. Foram realizadas observações de alguns espécimes de determinadas espécies com intuito de elucidar suas estruturas, através de Microscópio Óptico Olympus BH-2 (MO). Os desenhos foram realizados com auxílio de um tubo de desenho acoplado ao MO. Os espécimes representativos foram depositados na Coleção Helmintológica do Instituto Oswaldo Cruz - CHIOC, da Fundação Oswaldo Cruz, Rio de Janeiro, Brasil. Pelo menos um espécime de cada espécie de peixe foi depositado como hospedeiro representativo, simbiótipo sensu Brooks $^{12}$, na coleção do Instituto de Pesca, Santos, SP, e listados em Knoff et $\mathrm{al}^{13}$.

\section{RESULTADOS}

\author{
HOMEACANTHOIDEA Dollfus, 1942 \\ TENTACULARIIDAE Poche, 1926 \\ Heteronybelinia Palm, 1999
}

Heteronybelinia yamagutii (Dollfus, 1960) Palm, 1999

(Figuras 1-2)

Sinonímia: Nybelinia yamagutii Dollfus, 1960.

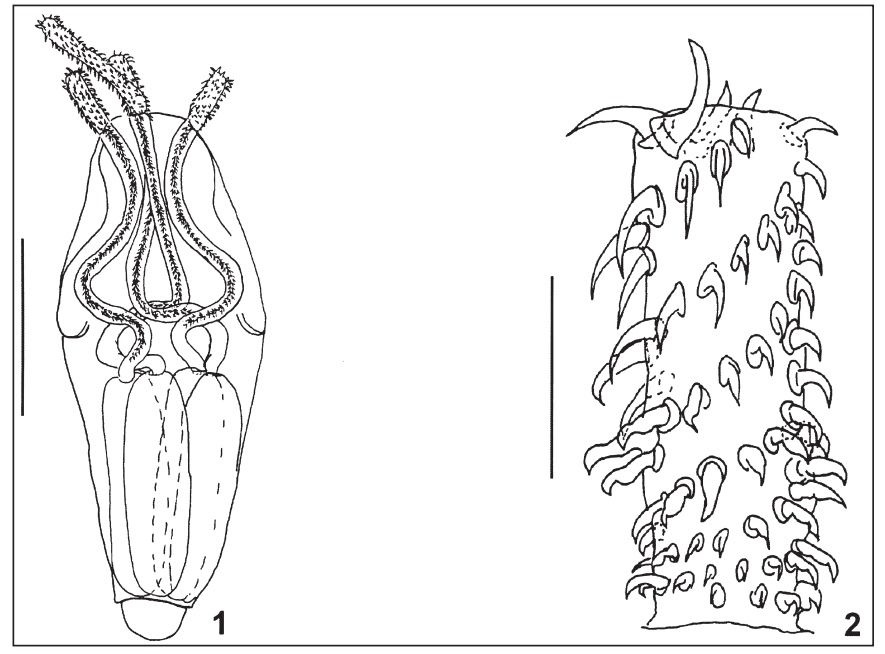

Figuras 1-2. Heteronybelinia yamagutii. 1. Escólex. 2. Tentáculo, região basal e debut da metabasal, face externa. Barra das figs. $1=1,0$ $\mathrm{mm} ; 2=0,125 \mathrm{~mm}$. 
Descrição do espécime baseada em 1 póslarva montada e medida, levemente comprimida. Escólex subcilíndrico com 3,56 de comprimento (incluindo velum, sem apêndice), maior largura do escólex ao nível da pars botridialis com 1,38. Pars botridialis apresentando botrídios pouco menores que a metade do comprimento do escólex com 1,64 de comprimento por 1,38 de largura. Pars vaginalis mais longa que a metade do escólex com bainhas levemente sinuosas, com 1,80 comprimento. Pars bulbosa com $1,48 \mathrm{de}$ comprimento por 0,86 de largura. Bulbos individuais longos com 1,43 $(1,40-1,46)$ de comprimento por $0,41(0,40-0,42)$ largura. Pars pós-bulbosa curta com 0,016 de comprimento. Apêndice curto com 0,24 de comprimento. Velum curto quase inexistente com $0,02 \mathrm{de}$ comprimento. Largura dos tentáculos sem ganchos, região basal com 0,104 , região metabasal com 0,108. Armadura dos tentáculos heteromorfa. Ganchos da região basal característicos, $1^{a}$ fileira: botridial com ganchos mais longos e de curvatura menos arqueada com 0,024 de comprimento por 0,012 de largura, antibotridial com ganchos mais curtos e recurvados, como uma garra, com 0,016 de comprimento por 0,010 a 0,012 de largura; $2^{a}$ fileira, tornam-se digitiformes apresentam este formato até a $6^{2}$ fileira: botridial continuam mais longos e de curvatura menos arqueada, com 0,024 de comprimento por 0,012 de largura, antibotridial continuam como uma garra com 0,016 de comprimento por 0,012 de largura; $3^{\mathrm{a}}$ e $4^{\mathrm{a}}$ fileira: botridial, mudança na ponta do gancho agora posicionada em um ângulo de $90^{\circ}$ para baixo, com 0,048 de comprimento por 0,016 de largura, antibotridial os ganchos apresentam a garra mais aberta, com 0,024 a 0,028 de comprimento por 0,012 de largura; $5^{\mathrm{a}}$ e $6^{\mathrm{a}}$ fileira: botridial ganchos com o mesma forma da $4^{\mathrm{a}}$ porém tornam-se mais retilíneos, com 0,060 a 0,064 de comprimento por 0,022 a 0,028 de largura, antibotridial os ganchos apresentando a ponta fortemente recurvada com 0,032 a 0,036 de comprimento por 0,012 de largura. Na região metabasal os ganchos perdem o formato digitiforme, as duas primeiras fileiras do início da metabasal: botridial com 0,054 a 0,072 de comprimento por 0,024 a 0,028 de largura, antibotridial com 0,028 a 0,036 de comprimento por 0,020 a 0,024 de largura; $13^{\mathrm{a}}$ fileira: botridial com 0,068 a $0,076 \mathrm{de}$ comprimento por 0,024 a 0,028 de largura, antibotridial com 0,040 de comprimento por 0,016 a 0,020 de largura. A armadura tentacular metabasal, na superfície antibotridial, consiste de ganchos espessos e fortemente recurvados e com uma base larga; na superfície botridial, a armadura tentacular metabasal consiste de ganchos mais delgados e levemente curvados no topo e com uma base encorpada, perdendo o aspecto digitiforme, pelo menos até as fileiras onde é possível se observar. Número de ganchos por fileira entre 16 e 18 . Todos tentáculos observados, parcialmente extrovertidos, só permitindo a observação de 16 fileiras de ganchos.

Hospedeiro/localização: 1 espécime na válvula espiral de 1 macho de Carcharhinus signatus (NH; NDG).

Proveniência: litoral do Estado do Paraná.

Espécime depositado: CHIOC n 34501 .

Comentários: As medidas do escólex como também a forma e tamanho da armadura tentacular do única pós-larva coletada correspondem as da descrição original de material proveniente de Dakar, Senegal ${ }^{2}$ como também de Moçambique, Namíbia e África do $\mathrm{Sul}^{4,8}$. Os espécimes coletados de cefalópodes de New England $^{6}$, costa atlântica dos EUA, apresentaram o escólex maior $(4,3$ - 5,0), mas, as demais estruturas do escólex e da armadura tentacular correspondem às da descrição original. Somente outras duas espécies conhecidas do gênero Heteronybelinia têm armadura basal característica constituida por ganchos digitiformes, $H$. rougetcampanae e $H$. nipponica, entretanto $H$. yamagutii é facilmente distinguível das outras por apresentar o escólex maior, pars bulbosa tão longa quanto a pars botridialis e a pars vaginalis, e os ganchos botridiais na metabasal com as pontas recurvadas como garras. No único espécime examinado em microscopia óptica, não foi possível observar microtríquias na estrutura superficial do escólex, como já documentado para esta espécie e em outros tentaculariídeos ${ }^{14-16}$. O encontro em $C$. signatus representa um novo registro de hospedeiro elasmobrânquio, reforçando a idéia sobre a distribuição mundial desta espécie, sugerida por outros autores ${ }^{4-8}$.

Heteronybelinia nipponica (Yamaguti, 1952) Palm, 1999

(Figuras. 3-5) 1952

Sinonímia: Nybelinia nipponica Yamaguti,

Descrição do espécime baseada em uma pós- 




Figuras 3-5. H. nipponica. 3. Escólex. 4. Tentáculo, região metabasal, face interna e região apical, face botridial. 5. Tentáculo, região basal, face botridial e debut da metabasal, face externa. Barra das figs. $3=0,5 \mathrm{~mm} ; 4-5=0,1$ $\mathrm{mm}$. larva, montada e medida, levemente comprimida. Escólex subcilíndrico com 0,80 de comprimento, maior largura do escólex ao nível da pars botridialis com 0,52 (incluindo velum, sem apêndice). Pars botridialis apresentando botrídios levemente elípticos com 0,54 de comprimento por 0,52 de largura. Pars vaginalis mais longa que a metade do escólex com bainhas sinuosas com 0,62 de comprimento. Pars bulbosa com 0,26 de comprimento por 0,23 de largura. Bulbos individuais com $0,16(0,15-0,18)$ de comprimento por $0,074(0,072-0,080)$ de largura. Pars pósbulbosa curta com 0,012 de comprimento. Apêndice com 0,18 de comprimento. Velum curto com 0,008 de comprimento. Largura dos tentáculos sem ganchos, região basal com 0,038. Região metabasal com 0,040. Armadura dos tentáculos heteromorfa. Ganchos da região basal característicos, $1^{2}$ fileira: botridial com ganchos mais alongados e de curvatura menos arqueada com 0,006 de comprimento por 0,002 de largura, antibotridial com ganchos mais curtos e recurvados, como uma garra, com 0,007 de comprimento por 0,003 de largura; $2^{a}$ fileira: tornam-se digitiformes, apresentam este formato até a 6 fileira: botridial, continuam mais alongados e de curvatura menos arqueados e a ponta do gancho posicionado em um ângulo de $90^{\circ}$ para baixo, com 0,008 a 0,010 de comprimento por 0,002 a 0,004 de largura, antibotridial, continuam como uma garra, com 0,007 de comprimento por 0,003 de largura; $3^{\mathrm{a}} \mathrm{e} 4^{\mathrm{a}}$ fileira: botridial, com 0,016 a 0,026 de comprimento por 0,004 a 0,008 de largura, antibotridial os ganchos apresentam a garra mais aberta, com 0,014 a 0,024 de comprimento por 0,004 a 0,007 de largura; $5^{\mathrm{a}} \mathrm{e}$ 6 fileira: botridial ganchos com o mesma forma da $4^{\mathrm{a}}$ porém tornam-se mais retilíneos, com 0,026 a 0,028 de comprimento por 0,008 de largura, antibotridial os ganchos começam a mudar a forma digitiforme para arqueada apresentando o meio do gancho fortemente recurvado com 0,022 de comprimento por 0,010 de largura. Na região metabasal os ganchos perdem o formato digitiforme que agora só é, levemente, notado nos botridiais, que permanecem ainda com a ponta em $90^{\circ}$, este tipo de ponta anguloso é visível até a $22^{\mathrm{a}}$ e última fileira extrovertida observada. Ganchos nas duas primeiras fileiras do início da metabasal: botridial de formato mais arqueado, com 0,022 a 0,024 de comprimento por 0,012 de largura, antibotridial com 0,016 a 0,018 de comprimento por 0,010 de largura; $12^{\mathrm{a}}$ e $13^{\mathrm{a}}$ fileira: botridial com 0,028 a 0,032 de comprimento por 0,013 a 0,014 de largura, antibotridial com 0,024 a 0,026 de comprimento por 0,012 de largura; a partir da 16 fileira os ganchos botridiais tornamse mais alongados e retilíneos, os antibotridiais mais abertos em sua curvatura; $20^{\mathrm{a}}$ a $22^{\mathrm{a}}$ fileira: botridial com 0,032 de comprimento por 0,009 a 0,010 de largura, antibotridial com 0,026 a 0,028 de comprimento por 0,012 de largura. A armadura tentacular metabasal, na superfície antibotridial, consiste de ganchos espessos e fortemente recurvados e com uma base larga; na superfície botridial, a armadura tentacular consiste de ganchos mais delgados e fortemente curvados no topo e com uma base encorpada, alterando 
suavemente o formato digitiforme. Número de ganchos por fileira com 10 na basal e 12 na metabasal. Tentáculos parcialmente extrovertidos.

Hospedeiro/localização: 1 espécime na válvula espiral de 1 macho de Carcharhinus signatus (NH; NDG).

Proveniência: litoral do Estado do Paraná.

Espécime depositado: $\mathrm{CHIOC} \mathrm{n}^{\circ} 34502$.

Comentários: A pós-larva coletada de $C$. signatus está de acordo com as medidas apresentadas pelos menores exemplares da descrição original ${ }^{1}$. O espécime coletado apresentou a pars vaginalis enovelada, característica não observada por Yamaguti. A armadura tentacular dos ganchos da basal e da metabasal correspondem a descrição original. Esta é a menor das três espécies do gênero Heteronybelinia que apresentam armadura basal tentacular com ganchos caracteristicamente digitiformes, facilmente distinguível das outras duas, $H$. rougetcampanae e H. yamagutii, por apresentar ganchos do ínicio da metabasal fortemente recurvados, os ganchos botridiais da metabasal mantendo a ponta recurvada com angulos em $90^{\circ}$ voltadas para baixo como apresentada nos ganchos da basal e bulbos quase duas vezes menores do que a pars botridialis. No único espécime examinado em microscopia óptica, não foi possível observar microtríquias na superfície do escólex. A presença em $C$. signatus representa um novo registro de hospedeiro e amplia o conhecimento sobre a distribuição da espécie no mundo, suportando a distribuição trans-oceânica, como já indicado para outros tentacularí́deos ${ }^{4,11,17}$.

Mixonybelinia Palm, 1999

Mixonybelinia beveridgei (Palm, Walter, Schwerdtfeger \& Reimer, 1997) Palm, 1999 (Figuras 6-8)
Sinonímia: Nybelinia beveridgei Palm, Walter, Schwerdtfeger \& Reimer, 1997.

Descrição dos espécimes baseada em 4 póslarvas, todos montados e medidos, levemente comprimidos. Escólex acraspédoto subcilíndrico com 5,68 (5,14-6,30) de comprimento (incluindo velum, sem apêndice), maior largura do escólex ao nível da pars botridialis 3,24 (3,063,32). Pars botridialis, botrídios variando em comprimento de um pouco menores a um pouco maiores do que o escólex, com 2,91 $(2,32-3,44)$ de comprimento por 3,24 (3,06-3,32) de largura. Pars vaginalis, variando no comprimento de, aproximadamente, metade a mais longa que a metade do escólex, com bainhas levemente sinuosas, às vezes retilínias, com 3,03 $(2,54-3,52)$ de comprimento. Anel ao redor da base da bainha do tentáculo. Pars bulbosa com 1,39 (1,20-1,50) de comprimento por $2,19(2,08-2,48)$ de largura. Bulbos individuais com 1,31 (1,14-1,45) de comprimento por $0,42(0,32-00,50)$ de largura. Pars pós-bulbosa com 0,48 de comprimento. Apêndice com 0,76 $(0,66-0,86)$ de comprimento. Velum com 0,66 (0,60-0,72) de comprimento. Largura dos tentáculos sem ganchos, região basal com $0,14(0,12-0,15)$, região metabasal com 0,12 $(0,10-0,13)$. Armadura dos tentáculos heteromorfa. Os ganchos da base são homeomorfos com uma base encorpada, uma haste esguia, e fortemente recurvados no topo com $0,027(0,020-0,032)$ de comprimento, base de implantação com 0,017 $(0,012-0,020)$ de largura. Região metabasal os ganchos aumentam de comprimento, com 0,062 (0,058-0,068), base de implantação com 0,024 $(0,022-0,028)$ de largura. Número de ganchos por fileira 14 a 16. Nas superfícies dos tentáculos das regiões metabasal botridial e antibotridial os
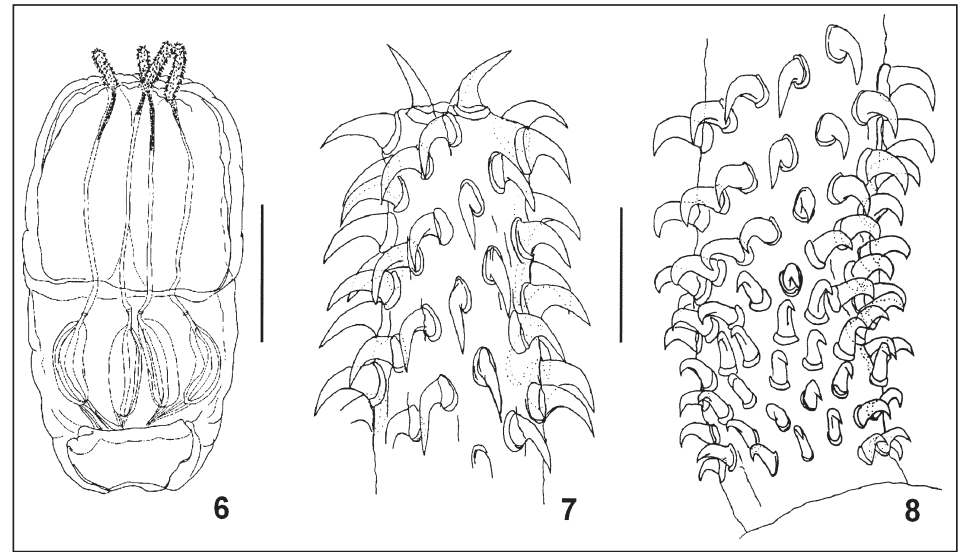

Figuras 6-8. Mixonybelinia beveridgei. 6. Escólex. 7. Tentáculo, região metabasal, face botridial a esquerda. 8 . Tentáculo, região basal, face botridial. Barra das figs. $6=2,0 \mathrm{~mm} ; 7-8=0,1$ $\mathrm{mm}$. 
ganchos apresentam diferenças no formato e tamanho, assim na antibotridial, a armadura tentacular consiste de ganchos espessos e fortemente recurvados e com uma base larga e na botridial, a armadura tentacular consiste de ganchos mais delgados e levemente curvados no topo e com uma base encorpada.

Hospedeiro/localização: 4 espécimes no estômago de 1 macho de Dipturus trachydermus (NH; NDG).

Proveniência: do litoral do Estado do Paraná.

Espécimes depositados: CHIOC n 34503 a-c.

Comentários: As pós-larvas coletadas de $D$. trachydermus, do litoral do Paraná, correspondem a descrição original ${ }^{8}$. Nos quatro espécimes examinados em microscopia óptica, não foi possível observar microtríquias na estrutura superficial do escólex. Dipturus trachydermus representa um novo registro de hospedeiro e amplia o conhecimento sobre a distribuição da espécie.

\section{RESUMO}

Espécimes de peixes elasmobrânquios, capturados em 1998 no Estado do Paraná, Brasil, estavam parasitados com cestóides Trypanorhyncha/Homeacanthoidea: Carcharhinus signatus com Heteronybelinia yamagutii e $H$. nipponica; Dipturus trachydermus com Mixonybelinia beveridgei Das duas espécies de Heteronybelinia foram obtidos detalhes da morfologia do escólex, que expandiram suas descrições. A distribuição geográfica das espécies foi ampliada.

\section{REFERÊNCIAS}

1.- YAMAGUTI S. Studies on the helminth fauna of Japan. Part 49. Cestodes of fishes, II. Acta Med Okayama 1952; 8: 1-76.

2.- DOLLFUS R P. Sur une collection de tetrarhynques homeacanthes de la famille de Tentaculariidae, récoltés principalement dans la région de Dakar. Bull Inst Fr Afr Noire 1960; 22(A): 788-852.

3.- SÃO CLEMENTE S C, GOMES D C. Description of the adult form of Nybelinia (Syngenes) rougetcampanae Dollfus, 1960 and some new data on $N$. (N.) bisulcata (Linton, 1889) (Trypanorhyncha: Tentaculariidae). Mem Inst Oswaldo Cruz 1992; 87: 251-5.

4.- PALM H W. Nybelinia Poche, 1926, Heteronybelinia gen. nov. and Myxonybelinia gen. nov. (Cestoda: Trypanorhyncha) in the collections of the Natural History Museum, London. Bull Nat Hist Mus (Zool Ser) 1999; 65: 133-53.

5.- GAEVSKAYA A V. Helminthofauna of Atlantic squid
Sthenoteuthis pteropus (Steenstrup). Nauckn Dokl Vyssh Shk Biol Nauki 1977; 8: 47-52. (Em Russo).

6.- STUNKARD H W. Studies on the tetraphyllidean and tetrarhynchidean metacestodes from squids taken on the New England coast. Biol Bull 1977; 153: 387-412.

7.- PASCUAL S, GONZÁLEZ A, ARIAS C, GUERRA A. Helminth infection in the short-fimred squid Illex coindetli (Cephalopoda, Ommastrephidae) off NW Spain. Dis Aquat Org 1995; 23: 71-5.

8.- PALM H W, WALTER T, SCHWERDTFEGER G, REIMER LW. Nybelinia Poche, 1926 (Cestoda: Trypanorhyncha) from the Moçambique coast, with description of $N$. beveridgei sp. nov. and systematic consideration on the genus. South Africa J Mar Sci 1997; 18: 273-85.

9.- AMATO J F R, BOEGER W A, AMATO S B. Protocolos para Laboratório - Coleta e Processamento de Parasitos de Pescado. Imprensa Universitária, Universidade Federal Rural do Rio de Janeiro, Seropédica, 81 p. 1991.

10.- CAMPBELL R A, BEVERIDGE I. Chapter 7.Order Trypanorhyncha Diesing, 1863. In: KHALIL L F, BRAY R A, JONES A (eds.). Keys to the cestode parasites of vertebrates. CAB, Cambtridge, United Kingdon, 751 p. 1994.

11.- DOLLFUS R P. Études critiques sur les tetrarhynques du Muséum de Paris. Arch Mus Natl Hist Nat (Paris) 1942; 19: 1-466.

12.- BROOKS D R. Extending the symbiotype concept to host voucher specimens. J Parasitol 1993; 79: 631-3.

13.- KNOFF M, SÃO CLEMENTE S C, PINTO R M, GOMES D C. Nematods of elasmobranch fishes from the southern coast of Brazil. Mem Inst Oswaldo Cruz 2001; 96: 81-7.

14.- PALM H W. Untersechungen zur Systematik von Rüsselbandwürmern (Cestoda: Trypanorhyncha) aus atlantischen Fischen. Ber Instit Meeresk Kiel 1995; 275: 233.

15.- PALM H W. Trypanorhynch cestodes from Indonesian coastal waters (East Indian Ocean). Folia Parasitol 2000; 47: 123-4.

16.- PEREIRA J R J. Trypanorhyncha (Cercomeromorphae, Eucestoda) nos Scianidae (Neopterygii, Perciformes) do litoral do Rio Grande do Sul: sistemática, estrutura das comunidades componentes e sua utilização como indicadores da estrutura trófica da assembléia hospedeira, DC Thesis, Universidade Federal do Paraná, Curitiba, 243 p. 1998.

17.- PALM H W, WALTER T. Nybelinia southwelli sp. nov. (Cestoda, Trypanorhyncha) with the redescription of $N$. perideraeus (Shipley \& Hornell, 1906) and synonymy of $N$. herdmani (Shipley \& Hornell, 1906) with Kotorella pronosoma (Stossich, 1901). Bull Nat Hist Mus (Zool Ser) 1999; 65: 123-31.

Agradecimentos: A programadora visual Heloisa Maria Nogueira Diniz do Laboratório de Produção e Processamento de Imagem Científica do IOC, FIOCRUZ, pela digitalização das figuras apresentadas. Ao Drs. Alberto Ferreira Amorim e Carlos Alberto Arfelli, do Instituto de Pesca de Santos, pelo apoio constante as viagens de pesquisa e identificação dos hospedeiros utilizados. A tripulação do barco de pesca Icanhema VI, pela ajuda na captura dos hospedeiros. Ao Conselho Nacional para Desenvolvimento da Pesquisa - CNPq, pelo suporte financeiro parcial. 